\title{
TWO TYPES OF HYPERINVARIANT SUBSPACES
}

\author{
ROBERT M. KAUFFMAN
}

\begin{abstract}
Let $A$ be a bounded operator in a Banach space $B$. Suppose that $A$ has the single valued extension property. Given a closed set $F$ in the complexes, define $\sigma_{A}(F)$ to be the set of all $x$ in $B$ such that there is an analytic function $x(\lambda)$ from the complement of $F$ to $B$ with $(A-\lambda I) x(\lambda)=x . A$ is said to have property $Q$ if $\sigma_{A}(F)$ is a closed subset of $B$ for every $F$.

Let $A$ be, again, a bounded operator in a Banach space $B$. Given a real number $b$, define $S_{A}(b)$ to be the set of all $x$ in $B$ such that $\exp (-c t) \exp (A t) x$ is a bounded function from the nonnegative reals to $B$ for all $c>b$. $A$ is said to have property $\mathrm{P}$ if $S_{A}(b)$ is a closed subspace of $B$ for all $b$.

These two properties are discussed in this paper.
\end{abstract}

Two types of closed invariant subspaces for a bounded operator $A$ are the subject of this paper. One is related to the solution of inhomogeneous equations; the other is related to the asymptotic behavior of $\exp (A t)$. Both are hyperinvariant or, in other words, invariant under all operators commuting with $A$.

We define two properties related to these types of invariant subspaces, which guarantee that if they occur they are closed. Property $\mathrm{Q}$ holds for all decomposable operators (see Colojoara and Foias [1]) and has been long known as one of the properties possessed by spectral operators.

In the sequel, $A$ will be taken to be a bounded linear operator from a Banach space $B$ into itself.

We first define property $\mathrm{Q}$. Suppose that $A$ has the single valued extension property, or in other words that there is no solution $x(\lambda)$ of the equation $(A-\lambda I) x(\lambda)=0$ for all $\lambda$ in some complex domain, such that $x(\lambda)$ is an analytic function from the domain to $B$. Define $\theta_{A}(x)$ to be the set of all $\lambda_{0}$ in the complexes such that the equation $(A-\lambda I) x(\lambda)=x$ is not solvable in any neighborhood of $\lambda_{0}$, with $x(\lambda)$ analytic. For a closed set $F$ in the complexes, define $\sigma_{A}(F)$ to be the set of all $x$ in $B$ such that $\theta_{A}(x)$ does not intersect the complement of $F$. We say that $A$ has property $\mathrm{Q}$ if $\sigma_{A}(F)$ is closed, for every closed set $F$, and $A$ has the single valued extension property. It is obvious that $\sigma_{A}(F)$ is a hyperinvariant subspace.

Received by the editors January 6, 1972.

AMS (MOS) subject classifications (1970). Primary 47A15.

Key words and phrases. Hyperinvariant subspace, single valued extension property, spectral operator, quasinilpotent operator, hyponormal operator.

(c) American Mathematical Society 1973 
We define property P. Let $c$ be a real number. Let $Q_{A}(c)$ be the set of all $x$ in $B$ such that $\exp (-c t)\|\exp (A t) x\|$ is a bounded function of $t$, where $t$ ranges over $[0, \infty)$. Given a real number $b$, the intersection of all $Q_{A}(c)$ with $c>b$ is denoted by $S_{A}(b)$. If $S_{A}(b)$ is closed for all $b$, then we say that $A$ has property $\mathrm{P}$.

It should be remarked that property $\mathrm{P}$ is defined in terms of $S_{A}(b)$ instead of $Q_{A}(b)$ in order that quasinilpotent operators have property $\mathrm{P}$. Clearly, if $A$ has property $\mathrm{P}$, all $S_{A}(b)$ are closed hyperinvariant subspaces for $A$.

LEMmA 1. If $x$ is in $S_{A}(b)$, then $\operatorname{Re}(\lambda) \leqq b$ for all $\lambda$ in $\theta_{A}(x)$.

Proof. For any $\lambda$ with $\operatorname{Re}(\lambda)>b$, define

$$
x(\lambda)=-\int_{0}^{\infty} \exp (-\lambda t) E(t) x d t, \quad \text { where } E(t)=\exp (A t) .
$$

It is not difficult to show that $(A-\lambda I) x(\lambda)=x$ for all $\lambda$ with $\operatorname{Re}(\lambda)>b$, and that $x(\lambda)$ is analytic on this domain.

THEOREM 1. If $A$ has property $\mathrm{Q}$ or property $\mathrm{P}$, and there is a point $\lambda_{0}$ such that $\lambda_{0}$ is in the spectrum of $A$, with $\operatorname{Re}\left(\lambda_{0}\right)>c$ and $S_{A}(c)$ nonempty, then $A$ has a nontrivial closed hyperinvariant subspace.

Proof. Let $x$ be in $S_{A}(c)$. By Lemma $1, \lambda_{0}$ is not in $\theta_{A}(x)$. However, if there is no $x_{0}$ such that $\lambda_{0}$ is in $\theta_{A}\left(x_{0}\right)$, then $\left(A-\lambda_{0} I\right)$ is surjective. In this case, $\lambda_{0}$ is in the point spectrum of $A$. The null space of $A-\lambda_{0} I$ is then a nontrivial closed hyperinvariant subspace. It is nontrivial because if $A=$ $\lambda_{0} I$, the hypotheses of the theorem cannot occur.

Thus we need only consider the case where $\lambda_{0}$ is in $\theta_{A}\left(x_{0}\right)$ for some $x_{0}$. By Lemma $1, x_{0}$ is not in $S_{A}(c)$, so that $S_{A}(c)$ is neither 0 nor $B$. If $A$ has property $\mathrm{P}$, then $S_{A}(c)$ is a nontrivial closed hyperinvariant subspace.

If $A$ has property $\mathrm{Q}$, let $R$ be an open ball about $\lambda_{0}$ in which the equation $(A-\lambda I) x(\lambda)=x$ is solvable, with $x(\lambda)$ analytic. (Recall that $x$ is in $S_{A}(c)$, so such a ball exists.) Let $R_{1}$ be the open ball about $\lambda_{0}$ with half the radius of $R$. Let $K$ be the complement of $R_{1} . x$ is in $\sigma_{A}(K)$, but $x_{0}$ is not. Thus $\sigma_{A}(K)$ is a nontrivial closed hyperinvariant subspace.

COROllary. If $A$ has a closed invariant subspace $S$ on which $\left\|A_{S}\right\|<$ $\operatorname{Re}\left(\lambda_{0}\right)$ for some $\lambda_{0}$ in a spectrum of $A$, then $A$ has a nontrivial closed hyperinvariant subspace, provided that $A$ has property $\mathrm{P}$ or property $\mathrm{Q}$. Here $A_{S}$ denotes the restriction of $A$ to $S$.

Proof. $S$ is invariant under $\exp (A t)$ and if $x$ is in $S,\|\exp (A t) x\| \leqq$ $\exp \left(\left\|A_{S}\right\| t\right)$. Thus $x$ is in $S_{A}(c)$, for any $c$ in between $\left\|A_{S}\right\|$ and $\operatorname{Re}\left(\lambda_{0}\right)$. By Theorem 1, the proof is finished. 
Lemma 2. Let $N$ be quasinilpotent, and suppose $N$ commutes with $A$. Then for any real number $b, x$ is in $S_{A}(b)$ if and only if $x$ is in $S_{A+N}(b)$.

Proof. Suppose $\exp (-c t) E(t) x$ is a bounded function of $t$ on $[0, \infty)$, where $E(t)=\exp (A t)$. Let $F(t)=\exp (N t)$. Then $E(t) F(t)=\exp ((N+A) t)$. However, for any $d>0,\|\exp (-d t) F(t)\|$ approaches zero as $t$ approaches infinity. The proof of this fact follows:

Note first that $\left\|N^{n}\right\|^{1 / n}$ approaches zero as $n$ approaches infinity, by the spectral radius formula. Recall that $F(t)=\sum_{0}^{\infty} N^{i} t^{i} / i$ !. Pick $J$ so large that $\left\|N^{n}\right\|^{1 / n}<d / 2$ for $n>J$.

Then

$$
\begin{aligned}
\|F(t)\| & \leqq \sum_{0}^{J}\|N\|^{i} t^{i} / i !+\left|\exp ((d / 2) t)-\sum_{0}^{J}(d / 2)^{i} t^{i} / i !\right| \\
& \leqq \sum_{0}^{J}\left(\|N\|^{i}+(d / 2)^{i}\right) t^{i} / i !+\exp ((d / 2) t) .
\end{aligned}
$$

Thus $\exp (-d t)\|F(t)\|$ clearly approaches zero as $t$ approaches infinity.

Now we finish the proof of the lemma. If $c>b$,

$$
\|\exp (-c t) E(t) F(t) x\| \leqq \exp (-a t)\|F(t)\| \exp (-(c-a) t)\|E(t) x\|,
$$

where $a=(c-b) / 2$. Thus if $x$ is in $S_{A}(b), \exp (-c t) E(t) F(t) x$ is a bounded function from $[0, \infty)$ to $B$ for $c>b$, so that $x$ is in $S_{A+N}(b)$. This completes the proof.

THEOREM 2. If $A$ has property $\mathrm{P}$, and $N$ is quasinilpotent and $N$ commutes with $A$, then $A+N$ has property $\mathrm{P}$.

REMARK. If $A$ has property $\mathrm{Q}$, and $N$ commutes with $A$, then $A+N$ has property Q. In fact, $\theta_{A}(x)=\theta_{A+N}(x)$ for all $x$. (See Colojoara and Foias $[1$, p. 17], for the proof of a more general proposition.)

THEOREM 3. Any spectral operator has property P.

REMARK. For the definition and properties of spectral operators, see Dunford and Schwartz [2, Part III]. As shown there, any spectral operator has property $\mathrm{Q}$.

Proof. By Theorem 2, we need only show that every scalar operator has property $\mathrm{P}$. To do this, we show that $S_{A}(b)=P(\theta)$, where $P(\theta)$ is the projection associated with the borel set $\theta$, and $\theta$ is the set of complex numbers with real part less than or equal to $b$.

Now, if $A$ is scalar, then by the functional calculus (or by direct computation) $\exp (A t)=\int \exp (\lambda t) d P_{\lambda}$ where $A=\int \lambda d P_{\lambda}$. (See Dunford and Schwartz [2, Part III, p. 1941].) 
Clearly, if $x$ is in $P(\theta) B$, $\exp (-\lambda t) \exp (A t) x$ is bounded for $\operatorname{Re}(\lambda)>b$, since by Corollary 4, Dunford and Schwartz [2, Part III, p. 1931], we know that the projection valued measure $P$ is bounded.

If $x$ is not in $P(\theta) B$, there is a such that $\Delta$ is an open subset of the complexes, the distance from $\Delta$ to $\theta$ is greater than $\varepsilon$, and $P(\Delta) x \neq 0$. Let $\lambda_{1}=b+\varepsilon / 2$. We will show that $\exp \left(-\lambda_{1} t\right) \exp (A t) x$ is unbounded, or in other words that $x$ is not in $S_{A}(b)$.

Call the bound for the projection valued measure $M$. Note that $\exp \left(-\lambda_{1} t\right) \exp A t=\exp (C t)$, where $C=\left(A-\lambda_{1} I\right)$. By the spectral decomposition for $A, \exp (-C t)=\int \exp \left(\lambda_{1} t-\lambda t\right) d P_{\lambda}$. Thus

$$
\|\exp (-C t) P(\Delta) x\| \leqq \exp (-t \varepsilon / 2) M\|P(\Delta) x\| .
$$

Also $\|P(\Delta) x\|=\|\exp (-C t) \exp (C t) P(\Delta) x\|=\|\exp (-C t) P(\Delta) \exp (C t) x\| \leqq$ $\exp (-t \varepsilon / 2)\|P(\Delta) \exp (C t) x\|$. Therefore $\|P(\Delta) \exp (C t) x\|$ approaches infinity with $t$, so the same must be true for $\|\exp (C t) x\|$. This completes the proof.

REMARK. It may be necessary to multiply an operator by a complex number in order to maximize the number or subspaces of type $S_{A}(b)$. For example, if $H$ is selfadjoint on a Hilbert space $h, S_{i H}(b)=h$ if $b \geqq 0$, and $S_{i H}(b)=0$ if $b<0$. However, $S_{H}(b)=P(\theta)$, where $P$ is the spectral measure associated with $H$ and $\theta$ is the borel set $[-\|H\|, b]$.

In general it is not clear to the author whether multiplication by a complex number destroys property $P$. In fact, it is doubtful whether the fact that $\boldsymbol{A}$ has property $\mathrm{P}$ implies that $-\boldsymbol{A}$ has property $\mathrm{P}$. Multiplication by a positive real number merely introduces a scale change and does not affect property $\mathbf{P}$.

The following theorem and corollary give some elementary observations related to the preceding remarks.

THEOREM 4. Suppose $c A$ has property $\mathrm{P}$ for every complex number $c$. Then the intersection of all $S_{\lambda A}(0)$, where $\lambda$ ranges over the complex numbers of modulus 1 , is a closed hyperinvariant subspace on which the restriction of $A$ is quasinilpotent.

Proof. Let $S$ be the subspace in question. It is clearly closed and hyperinvariant. By Lemma $1, \theta_{R}(x)=0$ for all $x$ in $S$, where $R$ is the restriction of $A$ to $S$. Thus the only nonzero points of the spectrum of $R$ are eigenvalues. Clearly, however, $R$ can have no nonzero eigenvalues from its definition. Thus $R$ is quasinilpotent.

COROLlaRY. Let $c A$ have property $\mathrm{P}$ for every complex number $c$. If there is a closed invariant subspace for $A$ on which the restriction of $A$ is quasinilpotent, then there is a maximal such subspace, and it is given by the subspace $S$ of Theorem 4. 
Proof. Any closed invariant subspace on which $A$ is quasinilpotent is contained in $S$, as can be deduced from Lemma 2 . Thus $S$ is the maximal such subspace, and the corollary is proved.

THEOREM 5. If $A$ has property $\mathrm{P}$ or property $\mathrm{Q}$, then the restriction of $A$ to any closed invariant subspace has the same property.

Proof. The case of property $\mathrm{P}$ is trivial, since any closed subspace invariant under $A$ is also invariant under $\exp (A t)$.

The case of property $\mathrm{Q}$ is more difficult. We must show that $\sigma_{R}(F)$ is closed for any closed set $F$ in the complexes, where $R$ is the restriction of $A$ to the closed invariant subspace $S$.

If $x_{n}$ approaches $x$, and $(R-\lambda I) x_{n}(\lambda)=x_{n}$ on the complement of $F$, with $x_{n}(\lambda)$ analytic, we must find an $x(\lambda)$ analytic on the complement of $F$ such that $(R-\lambda I) x(\lambda)=x$. By hypothesis we can solve the equation $(A-\lambda I) x(\lambda)=x$ with $x(\lambda)$ analytic on the complement of $F$. We will show that $x(\lambda)$ is in $S$ for every $\lambda$ in the complement of $F$.

Let $V$ be the topological vector space formed by the set of all analytic functions $y(\lambda)$ from the complement of $F$ into $B$, which have the property that $(A-\lambda I) y(\lambda)$ is a constant function from the complement of $F$ into $B$. Let the topology on $V$ be the topology of uniform convergence on the countable tower of open sets $\theta_{n}$, where $\theta_{n}$ is the set of all complex numbers $\lambda$ which are distant by more than $1 / n$ from $F$. Note that any element of $V$ is bounded on each $\theta_{n} . V$ is a Fréchet space. It is complete because, if $y_{n}$ is a Cauchy sequence in $V$, and $y$ is the pointwise limit, then $f(y(\lambda))$ is clearly an analytic function of $\lambda$ on the complement of $F$, if $f$ is in $B^{*}$. However, any weakly analytic function is analytic, by a theorem of Dunford (see Yosida [4, p. 128]).

The mapping $T(x(\lambda))=(A-\lambda I) x(\lambda)$ is a continuous one to one mapping from $V$ into $B$. Since $\sigma_{A}(F)$ is closed, and equal to the range of $T, T^{-1}$ must be continuous by the open mapping theorem. Therefore, if $(A-\lambda I) x_{n}(\lambda)=$ $x_{n}$ on the complement of $F$, and $x_{n}$ approaches $x$, then $x_{n}(\lambda)$ approaches $x(\lambda)$ in $B$ for each fixed $\lambda$. But in the case we deal with, $x_{n}(\lambda)$ is in $S$ for each $\lambda$. Therefore $x(\lambda)$ is in $S$ for each $\lambda$, and the theorem is proved.

THEOREM 6. If $A$ has property $\mathrm{P}$, then $A$ has the single valued extension property.

Proof. Suppose $(A-\lambda I) x(\lambda)=0$ for $x(\lambda)$ analytic in a ball $\theta$ about $\lambda_{0}$. Let $E(t)=\exp (A t)$. Then $E(t) x(\lambda)=\exp (\lambda t) x(\lambda)$. Thus $x(\lambda)$ is in $S_{A}(\operatorname{Re}(\lambda))$ for every $\lambda$ in $B$.

In particular, $S=S_{A}\left(\operatorname{Re}\left(\lambda_{0}\right)\right)$ is closed, and $x(\lambda)$ is in $S$ for every $\lambda$ in $\theta$ with $\operatorname{Re}(\lambda) \leqq \operatorname{Re}\left(\lambda_{0}\right)$. Let $f$ be a bounded linear functional on $B$ that annihilates $S$. Then $f(x(\lambda))$ is identically zero on the set of $\lambda$ in $\theta$ with 
$\operatorname{Re}(\lambda) \leqq \operatorname{Re}\left(\lambda_{0}\right)$. Since $f(x(\lambda))$ is an analytic function from $\theta$ into the complexes, $f(x(\lambda))$ must vanish identically on $\theta$. Thus any bounded linear functional which annihilates $S$ also annihilates all $x(\lambda)$ for $\lambda$ in $\theta$. By the Hahn-Banach theorem, $x(\lambda)$ must be in $S$ for all $\lambda$ in $\theta$. But this is impossible, since if $\lambda$ is in $\theta$ and $\operatorname{Re}(\lambda)>\operatorname{Re}\left(\lambda_{0}\right), x(\lambda)$ clearly cannot be in $S$.

Example. Let $A$ be an isometry in a Hilbert space. Then, as is well known, $A$ can be regarded as the restriction of a unitary operator in a larger Hilbert space. Therefore $A$ has property $\mathrm{P}$ and property $\mathrm{Q}$. However, if $A$ is not unitary, it can be shown (Colojoara and Foias [1, p. 10]) that $A^{*}$ does not have the single valued extension property, and thus does not have property $\mathrm{P}$ or property $\mathrm{Q}$.

Theorem 7. If $B$ is a Hilbert space, and $A$ is hyponormal, then $A$ has property $\mathrm{P}$.

Proof. Let $E(t)=\exp (A t)$. Then

$$
\begin{aligned}
& (E(t) x, E(t) x)^{\prime \prime}=\left(\left(A+A^{*}\right) E(t) x, E(t) x\right)^{\prime} \\
& \quad=\left(\left(2 A^{*} A+A^{2}+\left(A^{*}\right)^{2}\right) E(t) x, E(t) x\right) \geqq\left(\left(A+A^{*}\right)^{2} E(t) x, E(t) x\right) \geqq 0 .
\end{aligned}
$$

Recall that by definition $A$ is hyponormal if $A A^{*} \leqq A^{*} A$.

Thus if $\|E(t) x\|$ is bounded, $\left(\left(A+A^{*}\right) E(t) x, E(t) x\right) \leqq 0$ for all $t \geqq 0$, and conversely.

However, the set of all $x$ such that $\left(\left(A+A^{*}\right) E(t) x, E(t) x\right) \leqq 0$ for all $t \geqq 0$ is clearly closed in $B$. To sum up, if $A$ is hyponormal, $S_{A}(0)$ is closed in $B$.

But if $A$ is hyponormal, $(A-\lambda I)$ is hyponormal for all complex numbers $\lambda$. Since $\exp (A-\lambda I) t=\exp (-\lambda t) E(t)$, it follows that for any real number $b$, the set of all $x$ such that $\exp (-b t) E(t) x$ is a bounded function of $t$ on $[0, \infty)$ is closed in $B$. Therefore $S_{A}(b)$ is an intersection of closed sets, and is itself closed.

\section{REFERENCES}

1. I. Colojoara and C. Foias, Theory of generalized spectral operators, Math. and Appl., vol. 9, Gordon and Breach, New York, 1968.

2. (a) N. Dunford and J. T. Schwartz, Linear operators. I. General theory, Pure and Appl. Math., vol. 7, Interscience, New York, 1958. MR 22 \#8302.

(b) - Linear operators. II. Spectral theory. Selfadjoint operators in Hilbert space, Interscience, New York, 1963. MR 32 \#6181.

(c) - Linear operators. III. Spectral operators, Interscience, New York, 1971.

3. P. Fillmore, Notes on operator theory, Math Studies, no. 30, Van Nostrand Reinhold, New York, 1970. MR 41 \#2414.

4. K. Yosida, Functional analysis, 2 nd ed., Die Grundlehren der math. Wissenschaften, Band 123, Springer-Verlag, New York, 1968. MR 39 \#741.

Department of Mathematics, Western Washington State College, BellingHAM, WASHINGTON 98225 